\title{
A NOTE ON PAIRS OF NORMAL MATRICES WITH PROPERTY L
}

\author{
N. A. WIEGMANN
}

The following is a generalization of a theorem due to Motzkin and Taussky [1] concerning matrices with property L. By definition, two matrices $A$ and $B$ have property $\mathrm{L}$ if any linear combination $\alpha A+\beta B$ (where $\alpha$ and $\beta$ are complex numbers) has as characteristic roots the numbers $\alpha \lambda_{i}+\beta \mu_{i}$ where the $\lambda_{i}$ are the characteristic roots of $A$ and the $\mu_{i}$ are the characteristic roots of $B$ both taken in a special ordering. It is shown in the above paper that if two hermitian matrices have property $\mathrm{L}$, they commute.

The following lemma may be noted:

LEMMA. If a normal matrix has its characteristic roots in the main diagonal, then the matrix is diagonal.

Let $A$ be normal with characteristic roots $\alpha_{1}, \alpha_{2}, \cdots, \alpha_{n}$. Then there exists a unitary matrix $U$ such that $U A U^{C T}=D$ where $D$ is diagonal with the characteristic roots of $A$ appearing in the diagonal. Then $U A^{C T} U^{C T}=D^{C T}$ and $U A A^{C T} U^{C T}=D D^{C T}$ and the characteristic roots of $A A^{C T}$ are $\left|\alpha_{i}\right|^{2}$. Since the sum of the diagonal elements of $A A^{C T}$ is equal to the sum of its characteristic roots, all nondiagonal elements of $A$ must be zero if the characteristic roots of $A$ appear along the diagonal of $A$.

At this point the following theorem (Theorem 1) from [1] is recalled: Let the $n$-rowed square matrices $A$ and $B$ have property $\mathrm{L}$. Let $t$ be the number of different characteristic roots of $A$ and assume that all the characteristic roots $\lambda_{i}$ of $A$ are arranged in sets of equal roots. Let $m_{i}$ be the multiplicity of the characteristic root $\lambda_{i}$ of $A$ and assume there are $m_{i}$ independent characteristic vectors corresponding to each $\lambda_{i}$. Let $B_{1}=P^{-1} B P$ where $A_{1}=P^{-1} A P$ is in Jordan normal form. Then $B_{1}=\left(B_{i j}\right), i, j=1, \cdots, t$, where $B_{i i}$ is an $m_{i^{-}}$ rowed square matrix $(i=1, \cdots, t)$ and $\left|\lambda I-B_{1}\right|=\prod_{i=1}^{i}\left|\lambda I-B_{i i}\right|$.

ThEOREM. If $A$ and $B$ are normal matrices with property $L$, they commute.

Let the matrix $A$ be brought into diagonal form by an appropriate unitary matrix $U$ such that $U A U^{C T}=D$ is diagonal where like roots are grouped together. Let

Presented to the Society, December 28, 1951; received by the editors April 4, 1952. 


$$
U B U^{C T}=B_{1}=\left(B_{i j}\right)
$$

$(i, j=1, \cdots, k)$ where each $B_{i i}$ has an order equal to that of the number of like roots in the corresponding diagonal position in $D$. By the above-mentioned theorem the roots of $B_{1}$ are, in totality, the roots of $B_{11}, B_{22}, \cdots, B_{k k}$ taken together. From Schur [2] it is known that any square matrix with complex elements can be brought into a triangular matrix under a unitary transformation. Let $U_{i}$ be a unitary matrix such that $U_{i} B_{i i} U_{i}^{C T}=T_{i}$ where each $T_{i}$ is a triangular matrix. Let

$$
V=\left[\begin{array}{cccc}
U_{1} & 0 & \cdots & 0 \\
0 & U_{2} & \cdots & 0 \\
\vdots & & \ddots & \\
\dot{0} & 0 & & U_{k}
\end{array}\right]
$$

Then $V D V^{C T}=D$ and $V B_{1} V^{C r}$ is a normal matrix with the triangular matrices $T_{i}$ in the main diagonal and consequently its characteristic roots down the main diagonal. Hence $V B_{1} V C T$ is diagonal because of the lemma and it follows that $A$ and $B$ commute.

\section{BIBLIOGRAPHY}

1. T. S. Motzkin and O. Taussky, Matrices with Property L, Trans. Amer. Math. Soc. vol. 73 (1952) pp. 108-114.

2. I. Schur, Über die characteristischen Wurzeln einer linearen Substitutionen mit einer Anwendung auf die Theorie der Integralgleichungen, Math. Ann. vol. 66 (1909) pp. 488-510.

Washington, D. C. 